\title{
Composites on Base of the Ultradispersed Polytetrafluoroethylene and Graphite Oxide Intercalated Compounds
}

\author{
Vitaly I. Saldin, Alexander K. Tsvetnikov \\ Far-Eastern Branch of Russian Academy of Science, Institute of Chemistry, Vladivostok, Russia Federation \\ Email: sald@ich.dvo.ru
}

Received 9 November 2015; accepted 10 December 2015; published 17 December 2015

\begin{abstract}
Composites based on ultradispersed polytetrafluoroethylene and intercalated graphite oxide compounds with dodecahydro-closo-dodecaborates and methods of their fabrication have been developed. The fabricated composites have been characterized using XRD analysis, and optical microscopy. These composites are distinguished with completeness of their combustion, since the combustion products comprise gaseous boron fluorine-containing compounds of boron, boron trifluoride $\left(\mathrm{BF}_{3}\right)$, and boron oxyfluoride $\left((\mathrm{BOF})_{3}\right)$. Besides, these composites are characterized with increased energy capacity as compared to purely oxygen-containing compounds, since the heat of formation of boron fluorine-containing compounds is higher than that of boron oxide. Introduction of ultradispersed polytetrafluoroethylene imparts composites with hydrophobicity, thus improving their functioning properties.
\end{abstract}

\section{Keywords}

Composites, Ultradispersed Polytetrafluoroethylene, Graphite Oxide, Intercalated Compounds, Dodecahydro-Closo-Dodecaborates, Energy-Intensive Compositions

\section{Introduction}

Polytetrafluoroethylene (PTFE) is one of the most thoroughly studied polymers that has found rather extensive practical applications [1] [2]. In particular, PTFE-M (M-Al, Mg, Ti, $\mathrm{Zr}$ etc.) composites were suggested as mixed energy-rich powders [3]. They are produced using the method of combined grinding in ball mills. The phenomenon of transition of heavy polymer molecules into the gas phase in the process of PTFE thermal destruction with subsequent homophase nucleation and condensation in the form of nanofilms of a thickness of 2 $10 \mathrm{~nm}$ was discovered for the first time in the Institute of Chemistry FEBRAS in 1982. Depending on the condensation conditions, nanofilms can grow up to sizes of $100 \times 100 \mu \mathrm{m}$ and roll up into microtubes of a length of up to $150 \mu \mathrm{m}$ or a size of about $2 \times 2 \mu \mathrm{m}$ with subsequent formation of a pack of nanofilms as a microsphere of a diameter of about $1 \mu \mathrm{m}$ [4]. This material comprising a mixture of PTFE oligomers with the polymer chain length containing from $\approx 10^{1}$ to $10^{3} \mathrm{CF}_{2}$-groups was named ultradispersed polytetrafluoroethylene (UPTFE) [5]. 
A significant difference between UPTFE and PTFE (in the form of fluoroplast-4) is related to their powders particle sizes: $0.1-3.0 \mu \mathrm{m}$ and 160 - $200 \mu \mathrm{m}$, respectively. The latter fact opened new possible fields of practical application of UPTFE or significantly improved the available ones [6]. Equally important in fundamental and applied aspects is the graphite oxide (GO) [7] [8]. For example, fabrication of composites based on UPTFE and intercalated GO compounds with dodecahydro-closo-dodecaborate acid $\left(\mathrm{H}_{2} \mathrm{~B}_{12} \mathrm{H}_{12}\right)$, which are rather promising as energy-rich materials, can be performed through ultrasonic treatment of components aqueous solutions without the pre-pounding of fluoropolymer [9]. This is simpler and safer than application of mechanochemical methods [3]. In these composites, $\mathrm{H}_{2} \mathrm{~B}_{12} \mathrm{H}_{12}$ serves as a combustible, while combined UPTFE and GO-as an oxidizer. As fuel can be not only $\mathrm{H}_{2} \mathrm{~B}_{12} \mathrm{H}_{12}$, and its salts [10] [11].

The objective of the present work was to develop composites of UPTFE and IC of GO with $\mathrm{H}_{2} \mathrm{~B}_{12} \mathrm{H}_{12}$ or $\left(\mathrm{NH}_{4}\right)_{2} \mathrm{~B}_{12} \mathrm{H}_{12}$ and methods of their synthesis and study their properties.

\section{Experimental}

Studies of the process of thermal destruction of PTFE (fluoroplast-4) yielded the development of the thermal gas-dynamic (TGD) method and the technology of UPTFE production capable to create UPTFE manufacture on an industrial scale [12].

GO was obtained via the oxidation of natural graphite from the Zaval'ev deposit (Ukraine) using the modified Hummers-Offeman approach described in detail in [13]. After sedimentation of the reaction solution, the layer of clarified solution was decanted from the GO gel, and a fresh portion of distilled water was poured onto the sediment. After bringing the solution $\mathrm{pH}$ to 1 - 2, where the GO gel sedimentation rate dropped dramatically and the interface became diffuse, the solution was centrifuged on a K 70D centrifuge (Germany) at $7000 \mathrm{rpm}$. The GO gel was washed to $\mathrm{pH} \approx 5$ finally. To determine the content of the "dried" GO sample of his water-based gel was dried as a thin film at room temperature, and then milled into a powder and finely dried at $100^{\circ} \mathrm{C}$ until constant weight. To prevent the GO photoreduction a time-consuming operation of washing and drying the GO carried out in the dark. Analysis of "dried" GO into carbon and hydrogen was carried out by known methods of microanalysis of organic substances [14]. The composition of "dried" GO corresponds to the empirical formula $\mathrm{C}_{6} \mathrm{O}_{2,40} \mathrm{H}_{1,32} \times 0.58 \mathrm{H}_{2} \mathrm{O}$.

Potassium dodecahydro-closo-dodecaborate $\left(\mathrm{K}_{2} \mathrm{~B}_{12} \mathrm{H}_{12}\right)$ was obtained by pyrolysis of $\mathrm{KBH}_{4}-\mathrm{MBF}_{4}$ mixture (M-Na, K) according to [15]. The $\mathrm{B}_{12} \mathrm{H}_{12}{ }^{2-}$-anion separation from reaction products and its purification were performed using chitosan [16]. Salts of the $\mathrm{B}_{12} \mathrm{H}_{12}{ }^{2-}$-anion were obtained by treatment of chitosan dodecahydrocloso-dodecaborate $\left(\mathrm{C}_{6} \mathrm{O}_{4} \mathrm{H}_{9} \mathrm{NH}_{3}\right)_{2} \mathrm{~B}_{12} \mathrm{H}_{12}$ by respective hydroxides. To obtain the acid $\mathrm{H}_{2} \mathrm{~B}_{12} \mathrm{H}_{12}$, the cation exchange process of $\mathrm{M}_{2} \mathrm{~B}_{12} \mathrm{H}_{12}$ salts (M-Na) on the KU-2 ion exchange resin was used.

The content of the $\mathrm{B}_{12} \mathrm{H}_{12}{ }^{2-}$-anion in solutions of acid and ammonium salt was determined using the gravimetric method as $\mathrm{Ag}_{2} \mathrm{~B}_{12} \mathrm{H}_{12}$ [17].

IC synthesis was carried out through interaction of an aqueous gel of GO with aqueous solutions of $\mathrm{H}_{2} \mathrm{~B}_{12} \mathrm{H}_{12}$ and $\left(\mathrm{NH}_{4}\right)_{2} \mathrm{~B}_{12} \mathrm{H}_{12}$ at a molar ratio of 1 to $(0.1-0.3)$ [10] [11].

Optical images of the composite films were obtained with a microscope Axioplan 2 (Karl Zeiss, Germany).

The X-ray diffraction analysis was carried out on a DRON-3 and a D8 ADVANCE diffractometers (Bragg-Brentano method, $\lambda \mathrm{CuK}_{\alpha}$ ).

\section{Results and Discussion}

In the course of development of energy-active composites UPTFE-GO $\times \mathrm{nM}_{2} \mathrm{~B}_{12} \mathrm{H}_{12}\left(\mathrm{M}-\mathrm{H}, \mathrm{NH}_{4}\right)$, i.e., those that decompose the most completely, the following points were taken into consideration.

First, as was established earlier [18], heating of IC $\mathrm{GO} \times \mathrm{nH}_{2} \mathrm{~B}_{12} \mathrm{H}_{12}$ induced the exothermic reaction of intramolecular oxidation of dodecahydro-closo-dodecaborate fragments by oxygen present in the structure of GO. Here, the result is the formation of partially oxidized closo-dodecaborate anions $\mathrm{B}_{12} \mathrm{H}_{10} \mathrm{O}^{2-}$ self-organizing into the boron-polymer material $\left[-\mathrm{B}_{12} \mathrm{H}_{10} \mathrm{O}^{2-}-\right]_{\mathrm{n}}$ and the carbonaceous residue formed from the residual GO. ICs with high content of $\mathrm{M}_{2} \mathrm{~B}_{12} \mathrm{H}_{12}$ (M-H, Li, Na, K etc.) [19] were recommended as film-forming agents of water-based dispersion paints, whose drying yields thin coatings with high adhesion to the surface insoluble in water and a majority of known organic solvents and stable at heating in air up to $300^{\circ} \mathrm{C}$. It was demonstrated that at low $\mathrm{M}_{2} \mathrm{~B}_{12} \mathrm{H}_{12}$ content in IC, namely for compositions $\mathrm{GO} \times \mathrm{nM}_{2} \mathrm{~B}_{12} \mathrm{H}_{12}(\mathrm{n} \leq 0.3)$, the amount of oxygen in the 
structure of GO was sufficient for more complete oxidation of the $\mathrm{B}_{12} \mathrm{H}_{12}{ }^{2-}$-component. Heat emitted in this process warms up intermediate decomposition products up to temperatures sufficient for involving air oxygen into their complete oxidation. Decomposition of such ICs could proceed as an explosion or a flash upon fast heating or mechanical impact as in an inert atmosphere as in air [9] [10] [18].

Second, in development of such composites, one should take into account the ratio between UPTFE and IC. The ratio must ensure complete transformation of boron into boron oxyfluoride in air. It can be calculated from the following schemes:

$$
\begin{gathered}
3 \mathrm{nC}_{2} \mathrm{~F}_{4}+\mathrm{O} \Gamma \times \mathrm{nH}_{2} \mathrm{~B}_{12} \mathrm{H}_{12}+\mathrm{O}_{2} \rightarrow 4 \mathrm{n}(\mathrm{BOF})_{3}+\mathrm{H}_{2} \mathrm{O}+\mathrm{CO}_{2} \\
3 \mathrm{nC}_{2} \mathrm{~F}_{4}+\mathrm{O} \Gamma \times \mathrm{n}\left(\mathrm{NH}_{4}\right)_{2} \mathrm{~B}_{12} \mathrm{H}_{12}+\mathrm{O}_{2} \rightarrow 4 \mathrm{n}(\mathrm{BOF})_{3}+\mathrm{H}_{2} \mathrm{O}+\mathrm{CO}_{2}+\mathrm{N}_{2}
\end{gathered}
$$

The calculations show that in the case of UPTFE-GO $\times \mathrm{nH}_{2} \mathrm{~B}_{12} \mathrm{H}_{12}$ composites the fluoropolymer content must be equal to $19.6(\mathrm{n}=0.1), 30.4(\mathrm{n}=0.2)$, and $37.2 \mathrm{wt} \%(\mathrm{n}=0.3)$. In the case of UPTFE-GO $\times$ $\mathrm{n}\left(\mathrm{NH}_{4}\right)_{2} \mathrm{~B}_{12} \mathrm{H}_{12}$ composites, this value must be equal to $19.2(\mathrm{n}=0.1), 29.4(\mathrm{n}=0.2)$, and $35.7 \mathrm{wt} \%(\mathrm{n}=0.3)$. At the $\mathrm{M}_{2} \mathrm{~B}_{12} \mathrm{H}_{12}$ content increase in the composite, larger quantities of energy are emitted as a result of primary reaction of intramolecular interaction in IC GO. The latter results in more intensive heating of intermediate decomposition products that is sufficient for involvement of air oxygen and UPTFE into the process of complete oxidation. On the other hand, if the $\mathrm{M}_{2} \mathrm{~B}_{12} \mathrm{H}_{12}$ content increases, one has to increase the fraction of UPTFE in the composite in order to transform boron into boron oxyfluoride. In this case, the mass of products heated during primary interaction increases, so that the emitted heat could be insufficient to warm up intermediate decomposition products up to temperatures sufficient for involvement of air oxygen with attainment of complete combustion.

To sum up, to fabricate energy-active composites, it is necessary to determine experimentally the optimal ratio between their components (UPTFE, GO, and $\mathrm{M}_{2} \mathrm{~B}_{12} \mathrm{H}_{12}$ ), since respective calculations are rather complicated due to lack of thermodynamic data.

As was found earlier, to fabricate UPTFE-GO $\times \mathrm{nH}_{2} \mathrm{~B}_{12} \mathrm{H}_{12}$ composites [9], an intensive physical impact on the mixture of GO and UPTFE was used. It is related to the fact that UPTFE is characterized by expressed hydrophobic properties, and its introduction into the solution bulk is a very complicated task that is realized through powerful mechanical impact on UPTFE particles using two methods. The first one is concerned with using a high-speed stirrer (20,000 rpm), the second one-with ultrasonic impact. For instance, ingress of hydrophobic UPTFE particles into a narrow gap between rotating and stationary cylindrical shell rings of the stirrer results in their gradual activation due to emergence of dislocations, vacancies, shifts, and other defects in the polymer structure. As a result, particles become hydrophilic and move from the surface into the bulk of the GO aqueous gel. Similar modifications of UPTFE particles occur under effect of mixtures ultrasonic treatment as well.

The task can be significantly simplified by using ethanol dispersions of UPTFE, in which fluoropolymer particles are completely wetted by alcohol and do not float on its surface. Here, it was necessary to examine whether mixing of IC GO gel with UPTFE dispersion will result in gel destruction or $\mathrm{M}_{2} \mathrm{~B}_{12} \mathrm{H}_{12}$ salting-out from the IC GO composition.

As was established by the performed studies, mixing the aqueous gel of IC with the alcohol dispersion of UPTFE with the fluoropolymer content in the composite up to $40 \mathrm{wt} \%$ yielded the formation of homogeneous non-exfoliating gels. Upon their concentrating in air occurring mainly at the expense of ethanol evaporation and subsequent drying until the solid state, one does not observe chipping of UPTFE particles from the composite. According to the optical microscopy data, UPTFE particles are randomly distributed in the homogeneous film of IC (Figure 1). Composites diffractograms show the presence of PTFE and IC GO in them (Figure 2), which corroborates the absence of salting-out effect of ethanol on acid or ammonium salt and IC destruction. Upon ignition in air, all the fabricated composites explode or burn actively with formation of a light sooty residue. When conditioning in a humid atmosphere the weight gain of samples of the composite is not observed.

To sum up, composites based on UPTFE with intercalated compounds of GO with $\mathrm{M}_{2} \mathrm{~B}_{12} \mathrm{H}_{12}\left(\mathrm{M}-\mathrm{H}, \mathrm{NH}_{4}\right)$ have been developed. The optimal ratio between all the composite components has been found. A simple method of composite fabrication has been developed. The composites can be used as rather promising components of energy-rich materials of different functional applications. 


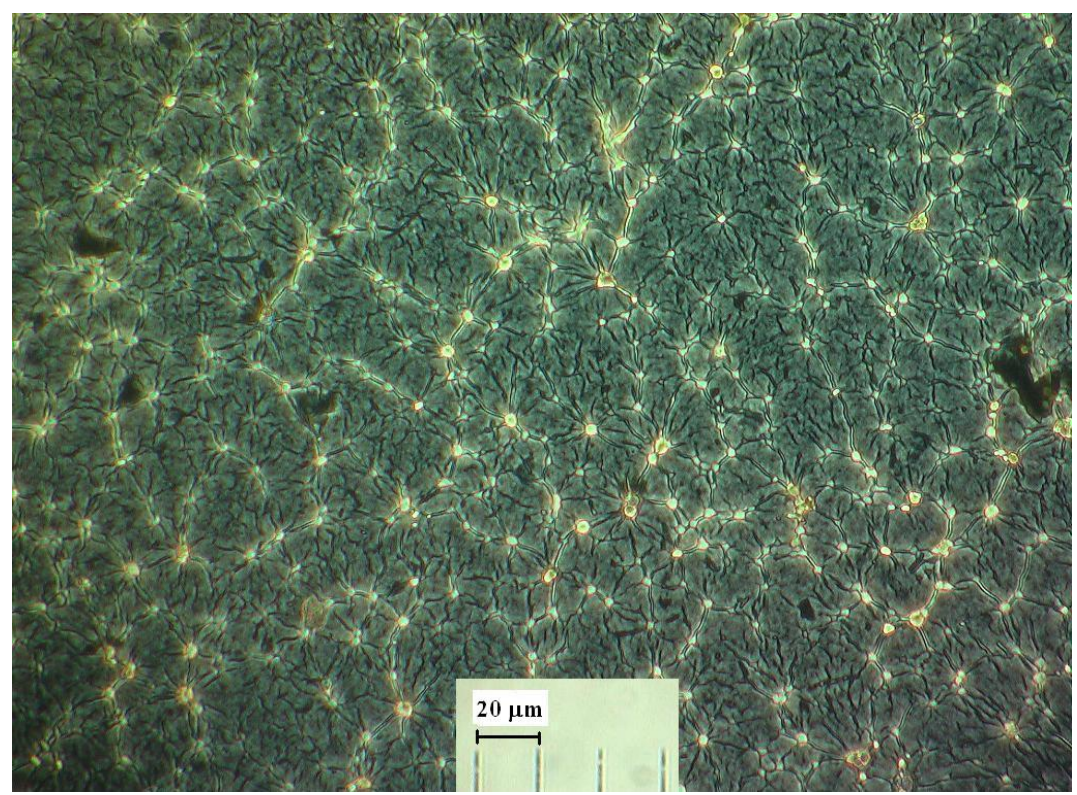

Figure 1. Optical image of the composite UPTFE-GO $\times 0.3 \mathrm{H}_{2} \mathrm{~B}_{12} \mathrm{H}_{12}$.

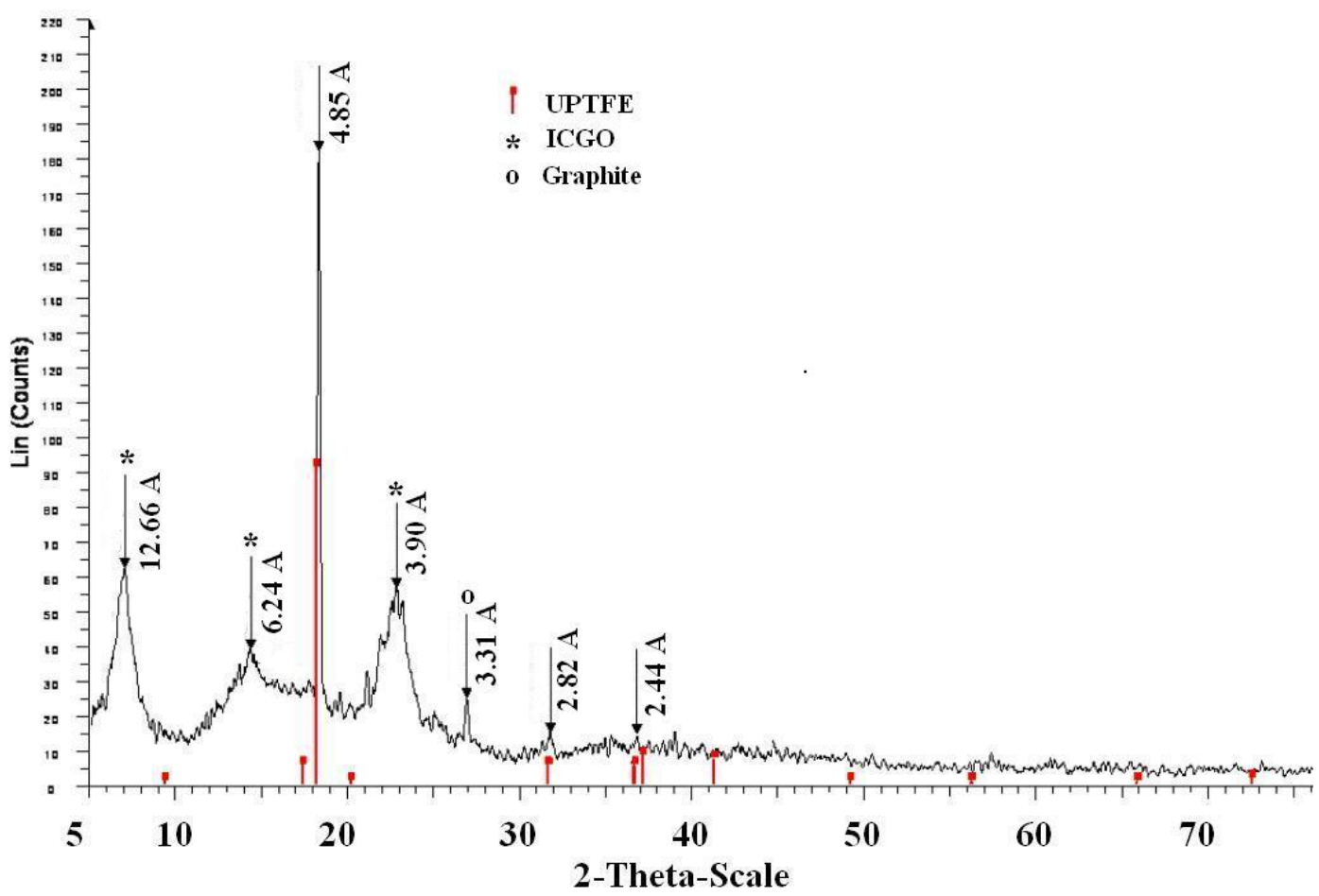

Figure 2. XRD of composite UPTFE-GO $\times 0.2\left(\mathrm{NH}_{4}\right)_{2} \mathrm{~B}_{12} \mathrm{H}_{12}$.

\section{Acknowledgements}

The work financially supported by the Program of Basic Research “”Far East” (project no. 265-2015-0011).

\section{References}

[1] Wall, L.A. (1972) Fluoropolymers. Wiley-Interscience, New York.

[2] Pashnin, Yu.A., Malkevich, S.G. and Dunaevskaya, Ts.S. (1975) Fluoroplastics, Khimia, L. 
[3] Dolgoborodov, A.Yu., Makhov, M.N., Kolbanev, I.V. and Fortov, V.E. (2006) The Pyrotechnic Composition Based on Mixtures of Mechanically Activated Metal-Oxidizer. Proceedings of III All-Russian Symposium Energetic Condensed Systems, Chernogolovka, 31 September-2 November 2006, 32-33.

[4] Tsvetnikov, A.K. and Uminskiy, A.A. (1990) A Method of Polyfluorocarbon Producing. RF Patent No 1808194.

[5] Pavlov, A.D., Sukhoverkhov, S.V. and Tsvetnikov, A.K. (2007) The Use of Pyrolytic Gas Chromatography/Mass Spectrometry to Determine the Composition of the Forum and Its Fractions. Bulletin of the Feb RAS, 51-55.

[6] Buznik, V.M. and Tsvetnikov, A.K. (1993) Ultradispersed Polytetrafluoroethylene as a Basis for New Advanced Materials. Bulletin of the Feb RAS, 39-47.

[7] Brodie, B.C. (1859) On the Atomic Weight of Graphite. Phil. Trans. R. Soc. London., 149, 249.

[8] Zhu, Y., Murali, S., Cai, W., et al. (2010) Graphene and Graphene Oxide: Synthesis, Properties, and Applications. Advance Materials, 22, 3906-3924. http://dx.doi.org/10.1002/adma.201001068

[9] Saldin, V.I. and Sukhovey, V.V. (2013) A Method of Producing of Boron-Fluorine-Containing Energy-Intensive Compositions. RF Patent No 2479560.

[10] Saldin, V.I., Tsvetnikov, A.K., Ignatieva, L.N. and Buznik, V.M. (2004) Intercalation of Graphite Oxide with Dodecahydro-Closo-Dodecaboric Acid. Russ. J. Inorg. Chem., 49, 1775-1779.

[11] Saldin, V.I. (2007) Regularity of Graphite Oxide Intercalation with Dodecahydro-Closo-Dodecaborate Anion. Russ. J. Inorg. Chem., 52, 1355-1358.

[12] Tsvetnikov, A.K. (1995) Installation for Processing of Polytetrafluoroethylene. RF Patent No 2035308.

[13] Saldin, V.I., Karpenko, A.A. and Tsvetnikov, A.K. (2010) Optical Properties of Coatings of Oxidized Graphite and Its Intercalated Compound with Ammonium Dodecahydro-Closo-Dodecaborate. Russ. J. Phys. Chem. A, 84, 1395-1400.

[14] Mazor, L. (1986) The Methods of Organical Analysis. Mir, Moscow.

[15] Saldin, V.I., Sukhovey, V.V., Buznik, V.M., Mikhailov Yu.M., Merkin, A.A. and Fedotov, P.I. (2010) A Method of Producing Chemical Compounds with Dodecahydro-Closo-Dodecaborate Anion. RF Patent No 2378196.

[16] Saldin, V.I., Sukhovey, V.V., Ignatieva, L.N., Slobodyuk, A.B., Buznik, V.M. and Mikhailov, Yu.M. (2010) Isolation of the Dodecahydro-Closo-Dodecaborate Anion with Chitosan from Aqueous Solutions. Theoretical Foundations of Chemical Engineering, 44, 467-470. http://dx.doi.org/10.1134/S0040579510040172

[17] Kuznetsov, N.T., Kulikova, L.N. and Kanaeva, O.A. (1976) The Gravymetric Determination of the Closo-Dodecaborates. Zhurnal Analytical Chem., 31, 1382-1383.

[18] Saldin, V.I., Tsvetnikov, A.K., Ignatieva, L.N., Nikolenko Yu.M. and Buznik, V.M. (2005) Study of Intramolecular Interaction in Intercalated Compounds of Graphite Oxide with Dodecahydro-Closo-Dodecaboric Acid by Heating. Russ. J. Inorg. Chem., 50, 1310-1315.

[19] Saldin, V.I. and Tsvetnikov, A.K. (2001) Intercalated Compounds of Graphite Oxide with Dodecahydro-Closo-Dodecaboric Acid and Its Salts, Their Use as Film-Forming Agent Water-Emulsion Paints and Method for Producing Polymer Coatings. RF Patent No 2165884. 